\title{
The crosstalk between the cardiovascular and the immune system
}

\author{
Carlo Dal Lin'1, Francesco Tona1 and Elena Osto ${ }^{2,3,4}$ \\ 1Department of Cardiac, Thoracic and Vascular Sciences, Padua University-Hospital, Padua, Italy \\ 2University and University Hospital Zurich, Institute of Clinical Chemistry, Zurich, Switzerland \\ 3University Hospital Zurich, Heart Center, Zurich, Switzerland \\ ${ }^{4}$ Swiss Federal Institute of Technology (ETH), Laboratory of Translational Nutrition Biology, Zurich, Switzerland
}

Correspondence should be addressed to C Dal Lin or E Osto: carlodallin@libero.it or elena.osto@uzh.ch

\begin{abstract}
The heart and the immune system are highly integrated systems cross-talking through cytokines, hormones and neurotransmitters. Their balance can be altered by numerous physical or psychological stressors leading to the onset of inflammation, endothelial dysfunction and tissue damage. Here, we review the main players and mechanisms involved in the field. A new research paradigm, which considers also novel contributors, like endothelial cells, is needed to better understand the pathophysiology of immune-mediated cardiovascular disorders and beyond.
\end{abstract}

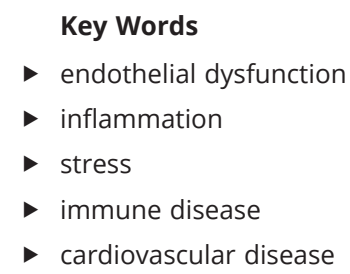

\section{Endothelial dysfunction, heart and immune system}

The endothelium is an active paracrine, endocrine, and autocrine organ (1) necessary for the regulation of the vascular tone and homeostasis. The endothelial function is considered a 'barometer' of cardiovascular health and is useful in prognosis evaluation and in therapeutic patient management $(2,3,4)$. In fact, not only cardiovascular risk factors $(2,3,4)$, but also several neuroendocrine and immunometabolic diseases switch the endothelial phenotype from health to disease-causing endothelial dysfunction (5). Indeed, the dysfunction of endothelial cells (ECs) represents the earliest key step in the development of most cardiovascular diseases. However, the molecular mechanisms that disrupt endothelial homeostasis remain unclear. Evidence indicates that, ECs are not simply bystanders lying between the circulating blood and the vessel wall components, but are the gatekeepers of a variety of signaling pathways actively controlling vascular integrity and exchanges between the circulation and different tissues and organs (6).
Moreover, once endothelial dysfunction arises, it can be regarded as a systemic condition (7) affecting the coronary as well as the peripheral vasculature.

A major determinant of endothelial health is the immune system. In fact, endothelial dysfunction is a hallmark in several immuno-mediated pathologies (i.e. vasculitis, myocarditis, thyroiditis, psoriasis, systemic lupus erythematosus, antiphospholipid syndrome, idiopathic inflammatory myositis, mixed connective tissue diseases, rheumatoid arthritis, systemic sclerosis, Sjögren's syndrome, graft disease after transplantation etc.) (6). The role of the innate immune system (monocytes and macrophages) together with neutrophils and lymphocytes (6) has also been well explored in relation to the onset and development of atherosclerosis. Interestingly, the electrical activity of the cardiac conduction tissue is also modulated by the immune system with a proven immunological component driving some rhythm and conduction disorders (8). Finally, after 
any insult the immune response may trigger either repair or regeneration processes in physiologic conditions or fibrosis in pathologic ones (6).

Nowadays, the heart itself is seen as an important immune station, able to fine-tune the function of the immune system by means of specific cardiokines (i.e. natriuretic peptides, tumor necrosis factor-alpha, transforming growth factor-beta, growth differentiation factor-15, activin-A, myostatin, adrenomedullin, and endocannabinoids) (1). It is becoming increasingly clear that a continuous flow of molecular information is exchanged between the cardiovascular and the immune system (9).

However, how the communication between the cardiovascular and the immune system takes place is not fully understood.

\section{The immune system beyond the fight against infections: the sense of danger}

Our understanding of the immune system has evolved over the past decades with the recognition that this is a dynamically regulated organ not only able to discriminate between 'self' and 'non-self' and fight against pathogenic microorganisms, but it plays a pivotal role in a variety of physio-pathological processes from organ development to tissue homeostasis and repair (6) (Fig. 1). Although not commonly considered part of the immune system, stromal and mesenchymal cells like fibroblasts and endothelial cells play crucial immunological functions. Moreover, the inflammatory status of the tissue environment is important in defining the type of response to any antigen and the immune system is crucial for the maintenance and restoration of tissue homeostasis in both sterile and infectious situations.

As we detailed elsewhere (2) a novel interpretation framework starts with the concept that the immune system recognizes as 'danger' all potential harms causing tissue stress or damage. According to this model, stressed tissues release alarm signals that activate antigen-presenting cells. This is the first check-point to activate immune responses. During the cellular response to stress (called 'universal cell danger response' (CDR), damaged cells and the immune system activate a sterile inflammation (or inflammasome)). Danger signals are recognized by the innate immune system through different receptors (pathogen recognition receptors (PRR) such as Tolllike receptors (TLR), nod-like receptors and receptors for advanced glycation end-products) that activate the production of cytokines and attract leukocytes to the site of lesion $(2,3,4)$. If the CDR persists abnormally beyond the injury resolution, the whole body metabolism and the gut microbiome will be disturbed, leading to multiple organ dysfunction and the onset of chronic diseases $(10,11,12,13,14,15,16)$. This phenomenon is accompanied by the activation of the psycho-neuroendocrine-immunitary (PNEI) network.

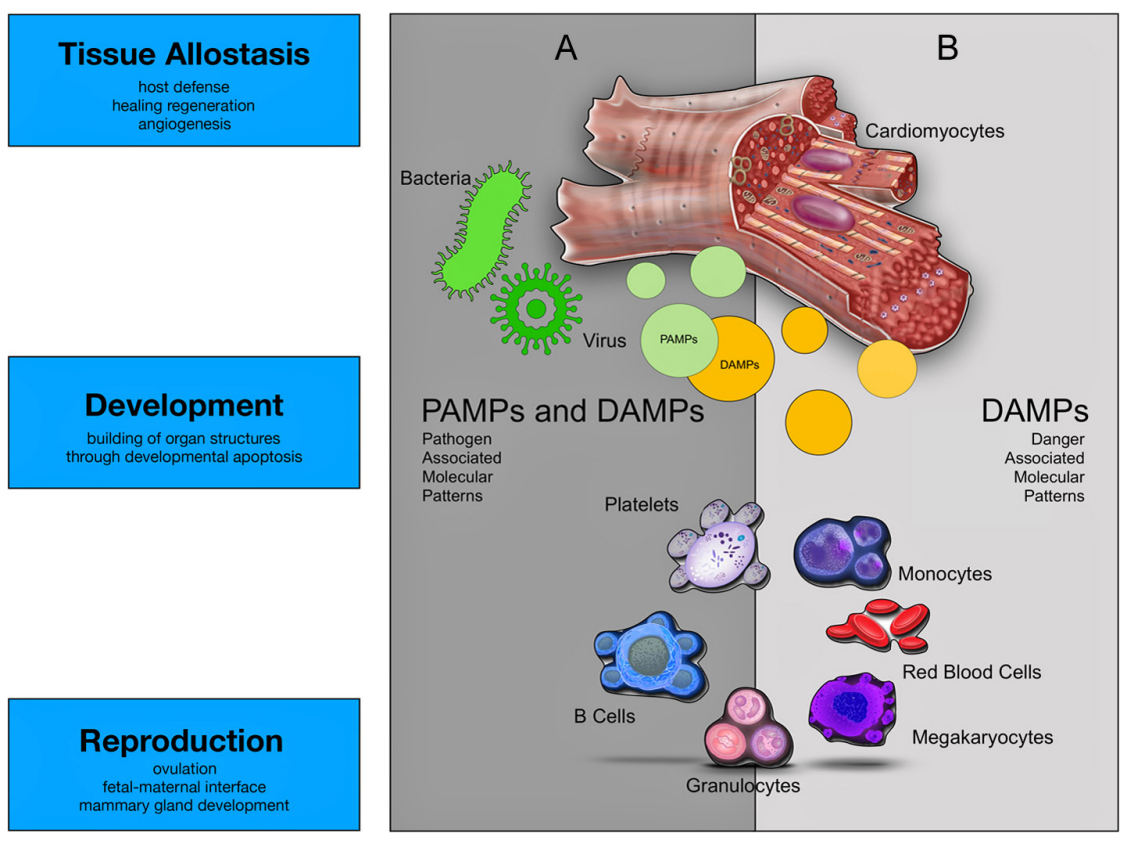

Figure 1

The figure depicts the fundamental roles of the immune system beyond host defense. (A) The immune system eliminates microbes in case of injury at barrier sites (e.g. skin) or primary infectious tissue damage (e.g. viral or bacterial myocarditis). (B) The immune system is essential for allostasis $(2,3,4)$, development and reproduction. Sterile tissue damage such as psychological (36) or physical trauma (37), mechanical stress (38) or ischemia/reperfusion injury (e.g. myocardial infarct) induces an inflammatory reaction promoting wound healing and/or regenerative mechanisms. Necrotic cells in damaged tissue release damage/dangerassociated molecular patterns (DAMPs) such as HMGB1, IL-33, ATP, heat-shock proteins, nucleic acids and ECM degradation products. Microbes are recognized by the immune system through their expression of pathogen-associated molecular patterns (PAMPs) such as LPS, flagellin, dsRNA and unmethylated motifs in DNA. ATP, adenosine triphosphate; ECM, extracellular matrix; HMGB1, high mobility group box 1 .

https://vb.bioscientifica.com https://doi.org/10.1530/VB-19-0023 (c) 2019 The authors Published by Bioscientifica Ltd

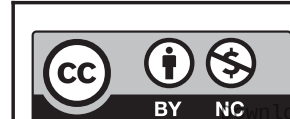

This work is licensed under a Creative Commons Attribution-NonCommercial 4.0 International License. ded from Bioscientifica.com at 04/26/2023 08:45:05AM 


\section{Cardiac myocytes, fibroblasts, endothelial cells, pericytes and cardiac tissue macrophages as a part of the 'innate immune system'}

Janeway described the existence of the 'innate immunity' as a simple, but rapid immune response (17), found in non-immunologic cell types and tissues. Indeed, the innate immunity is activated by the TLRs and is essential for rapid protection against infections. The downside of the innate response lies in its potential aspecific activation by proteins, RNA, and other endogenous ligands (called 'alarmins' or 'danger signals') that can lead to harmful cell and tissue inflammation/damage (6). Unfortunately, a number of essential and otherwise innocuous molecules activate different TLRs leading to inflammation accompanied by myocyte or ECs death/ injury and to organ dysfunction. Aberrant activation of TLR4 plays a significant role in cardiac injury. Other TLRs, including TLR2, seem also involved, although to a lesser extent (6).

Traditionally considered as non-immunologic cells, cardiac fibroblasts (CFs) are newly believed as the immunomodulatory hub of the heart (6). Under homeostatic conditions, immune cells and fibroblasts reside in the myocardial interstitium and sense microenvironmental cues maintaining the extracellular matrix in homeostatic conditions. Upon an injury, such as ischemic events or pressure overload, cardiac fibroblasts cross-talk with various immune cells and modulate the activity of both innate and adaptive immune systems (18). Indeed, after an injury, CFs undergo a transformation acquiring a myofibroblast phenotype and contribute to cardiac fibrosis. This remodeling causes well-known pathological changes like heart chamber dilation, cardiomyocyte hypertrophy and apoptosis leading to heart failure. Fibrosis is a crucial pathophysiological process in the development of cardiovascular disease. Current research aims to histologically localize and trace the origins and fate of CFs in various diseases in order to better understand the molecular mechanisms of $\mathrm{CF}$ activation. Among others the transforming growth factorbeta pathway, the angiotensin and endothelin system, the pathway of RhoA serum response factor myocardinrelated transcription factor but also the transient receptor potential channel network are emerging as fundamental players together with the connective tissue growth factor pathway, integrins and connexins networking and molecules of the adrenergic system. The crosstalk between these pathways and molecules seems to play an essential role in switching on the genetic machinery initiating profibrotic changes in the heart. Better understanding of these mechanisms will open new opportunities for development of targeted therapeutic approaches against cardiac fibrosis and its pathological contribution to disease progression $(19,20)$.

Another novel player is ECs. These cells communicate with immune cells through EC-secreted factors and endothelial dysregulation contributes directly to mediate immune responses at places of injury or infection (21). For instance, ECs are both culprits and victims during myocardial infarction. Although ECs tolerate hypoxia better than other cardiac resident cell types, their proangiogenic activation causes loss of barrier function and edema formation sustaining the cardiac immune cell infiltration. Accordingly, ECs pro-inflammatory activation increases the expression of adhesion molecules and causes leukocyte infiltration. Excessive immune cell infiltration can be detrimental to the already damaged tissue. Loss of nitric oxide (NO) synthesis by ECs impairs vascular dilatation and this further aggravates vessel occlusions in the heart. In addition, endothelial activation promotes a pro-thrombotic state and is able to activate the neuroendocrine hypothalamic-pituitary-adrenal D(HPA) stress axis (22). Thus, the loss of endothelialmediated immunomodulation needs to be considered as detrimental as the impairment of the other better known endothelial properties, such as angiogenesis, hemostasis, and the regulation of vascular tone (6).

The role of pericytes in disease has been described in many organs, and pericyte dysfunction has been implicated in tumor angiogenesis, atherosclerosis and Alzheimer's disease (6). Pericytes are a complex cell type playing many roles depending on the tissue they reside in (6). In addition to their well-established role in vascular formation, stabilization, remodeling, and function (pericytes transplantation techniques are even being tested to improve cardiac vasculature after myocardial infarction in animal models (23)), pericytes play a pivotal role in the immune response, attracting immune cells to the site of injury and regulating the migration of immune cells into the damaged tissue. Alterations of the fine-tuned interactions between pericytes and ECs, as documented in numerous genetic mouse models and certain human disease, result in severe and often lethal cardiovascular defects, diabetic microangiopathy, ectopic tissue calcification, stroke and dementia likely throughout the involvement of the transforming growth factor-beta pathway, TPBG (trophoblast glycoprotein) and the CXCL12/CXCR7/pERK axis (24), angiopoietins and

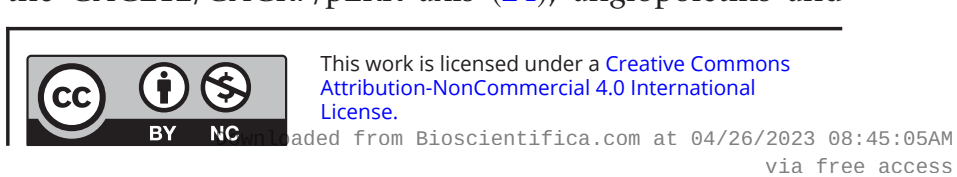




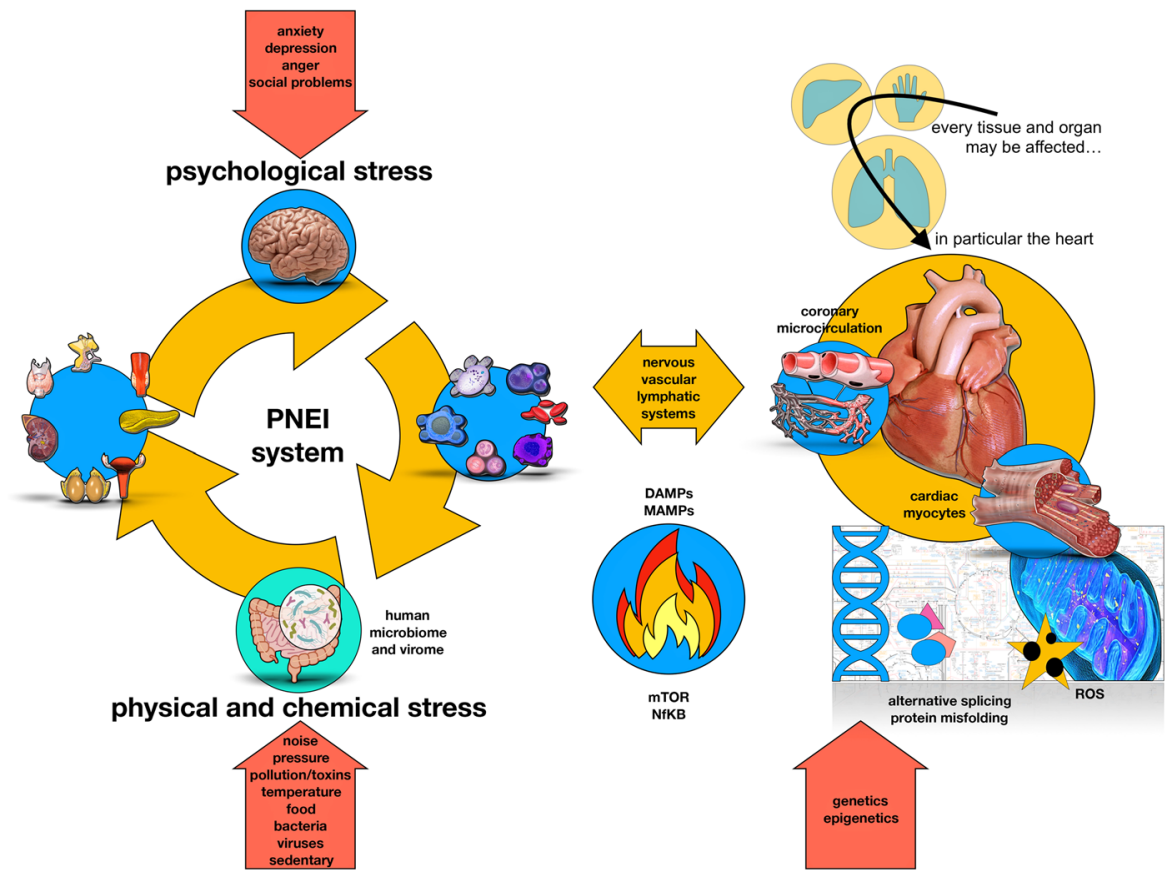

Figure 2

The human organism always tries to maintain its stability by adapting (allostasis) to external (physical and chemical) or internal (psychological) stimuli. In particular, stimuli are divided into physiological or non-physiological. Non-physiological stimuli (physical/chemical and psychological) are able to stress our psycho-neuro-endocrine-immune adaptation system (PNEI) (35) (left panel). Along with neurotransmitters, hormones and cytokines, also the human microbiome (and virome) plays an important role in the pathogenesis of many immune-mediated diseases, including cardiovascular disorders (29). The resulting immune activation can potentially affect all tissues. At the cardiovascular level, immune cells (activated either by a psychological or physical stressor) interact with endothelial cells, cardiac stromal cells and cardiomyocytes, which adapt their metabolism to support the ongoing inflammatory process (upper part right panel). In the heart (lower side of the right panel), the stress signal can mediate the activation of the intracellular transduction pathways linked to mTOR (39) and NfKB (40) genes through the mediators of the PNEl system (i.e hormones, neurotransmitters and cytokines). This will influence the cellular energy metabolism with high oxidative stress and damage from free radicals (41), misfolding of proteins (42) or their abnormal synthesis (alternative splicing) (43). Thus, cardiac cells expose danger signals that are detected by the immune system. The whole process can be resolved with regeneration/repair (depending on the tissue potential) in case of control and resolution of the acute stimulus or with tissue destruction in case of stress persistence and chronic processes. Of note, the 'cardioimmune' inflammatory activation can be activated by psychological stress and the stress activated patterns can be transmitted across generations via epigenetic modulation $(44,45)$.

angiocrine signaling (25), platelet-derived growth factor network, spingosine-1-phosphate and Notch ligands and their respective receptors $(26,27)$.

As described by Alexei Ilinykh and Alexander R Pinto (6), cardiac tissue macrophages (cTMs) are phagocytic cardiac resident leukocytes that are activated by danger-associated molecular patterns (DAMPs). These cells, in concert with fibroblasts, platelets, and ECs, release cytokines that initiate an inflammatory cascade and recruit circulating leukocytes into the injured tissue. During inflammatory responses cTMs differentiate into the 'M1' or 'classically activated' macrophages and secrete proteolytic and inflammatory agents (such as IL- $1 \beta, \mathrm{TNF} \alpha, \mathrm{NO}$, and IL-6). Later on, pro-inflammatory macrophages are replaced by macrophages with an anti-inflammatory phenotype (commonly termed 'alternatively activated' or ' $\mathrm{M} 2$ ') that help healing resolution of the lesion. Interestingly, it has been recently demonstrated (28) that macrophages may be implicated in normal and aberrant cardiac electrical conduction. In fact, cardiac macrophages seem to promote electrical conduction in the atrioventricular node through connexin 43-gap junctions. On the other hand, deletion of connexin 43 in macrophages or congenital lack of macrophages may delay the atrioventricular conduction and even cause an atrioventricular block.

Thus, cardiac myocytes, fibroblasts, ECs, pericytes and cTMs continuously exchange molecular/electrochemical information with the immune system keeping the cardiovascular and neuroendocrine-immune system in equilibrium (29). Indeed, stress of different nature can alter the cardio-immuno homeostasis with potential diseases onset (Fig. 2). 


\section{Psychic activity may modulate the crosstalk between the immune system and the heart and should be considered in the study of endothelial function and 'cardio-immuno' diseases}

The same inflammatory pathways briefly described in Figs 1 and 2 can also be activated in a 'sterile' way by mental stress (30). In fact, mental stress can lead to cellular damage with exposure of alarm molecules (i.e. beta 2 adrenergic receptors, protein kinase A, vascular endothelial growth factor, matrix metal proteinase- 2 and 9, S-100A1-9 proteins, DAMPs (heat shock protein-72, HMGB1, proteins RAE-1 and Mult1)) and the subsequent immune reaction may proceed toward damage and fibrosis. Current evidence closely links the psychological/ emotional profile of a subject with inflammation and harmful effects, like cardiac tissue damage (31). Myocardial ischemia induced by mental stress and the 'happy heart' or 'Tako-tsubo' syndrome are now well-defined diseases (1). Indeed, we have recently described (32) how proper stress management (for example through meditation or music appreciation) can reduce inflammation. Hence, reduced cytokines and lower expression of NFkB and TLR-4 receptor in peripheral blood mononuclear cells (PBMCs) contributed together with neuroendocrineimmune mediators and miRNAs to improved coronary function (33).

\section{A conclusive consideration}

A deeper understanding of cardiovascular immunology and in general of systems biology (34) need a more comprehensive framework taking into account genetic, but also environmental factors and the close relationship existing between the environment, psyche and the neuroendocrine system (35). We need to evolve our approach to understand how single threads compose a sophisticated embroidery.

\section{Declaration of interest}

The authors declare that there is no conflict of interest that could be perceived as prejudicing the impartiality of this review.

\section{Funding}

The continued financial support by the Swiss National Science Foundation Ambizione and Prima Grant (PZ00P3_161506 and PRO0P3_179861/1 to E O) and the Swiss Card-Onco-Grant - Alfred and Annemarie von Sick Grant (to E O) are gratefully acknowledged.

\section{Author contribution statement}

C D L conceived and drafted the manuscript and figures; gave final manuscript approval; agrees to be accountable for all aspects of work ensuring integrity and accuracy. F T and E O conceived and made critical revision of the contribution for important intellectual content, gave final manuscript approval; agree to be accountable for all aspects of work ensuring integrity and accuracy.

\section{References}

1 Dal Lin C, Tona F \& Osto E. The heart as a psychoneuroendocrine and immunoregulatory organ. Advances in Experimental Medicine and Biology 20181065 225-239. (https://doi.org/10.1007/978-3-319-77932-4_15)

2 Dal Lin C, Poretto A, Scodro M, Marra MP, Iliceto S \& Tona F. Coronary microvascular and endothelial function regulation: crossroads of psychoneuroendocrine immunitary signals and quantum physics [Part A]. Journal of Integrative Cardiology $2015 \mathbf{1}$ 132-163. (https://doi.org/10.15761/JIC.1000135)

3 Dal Lin C, Poretto A, Scodro M, Marra MP, Iliceto S \& Tona F. Coronary microvascular and endothelial function regulation:crossroads of psychoneuroendocrine immunitary signals andquantum physics [Part B]. Journal of Integrative Cardiology 20151 164-188. (https://doi.org/10.15761/JIC.1000136)

4 Dal Lin C, Poretto A, Scodro M, Marra MP, Iliceto S \& Tona F. Coronary microvascular and endothelial function regulation:crossroads of psychoneuroendocrine immunitary signals andquantum physics [Part C]. Journal of Integrative Cardiology 20151 189-209. (https://doi.org/10.15761/JIC.1000137)

5 Dal Lin C, Tona F \& Osto E. Coronary microvascular function and Beyond: the crosstalk between hormones, cytokines, and neurotransmitters. International Journal of Endocrinology $2015 \mathbf{2 0 1 5}$ 312848. (https://doi.org/10.1155/2015/312848)

6 Sattler S \& Kennedy-Lydon T. The Immunology of Cardiovascular Homeostasis and Pathology. Springer, 2017. (https://doi. org/10.1007/978-3-319-57613-8)

7 Anderson TJ, Gerhard MD, Meredith IT, Charbonneau F, Delagrange D, Creager MA, Selwyn AP \& Ganz P. Systemic nature of endothelial dysfunction in atherosclerosis. American Journal of Cardiology 199575 71B-74B. (https://doi.org/10.1016/00029149(95)80017-m)

8 Maisch B \& Ristic AD. Immunological basis of the cardiac conduction and rhythm disorders. European Heart Journal 200122 813-824. (https://doi.org/10.1053/euhj.2000.2186)

9 Pober JS \& Tellides G. Participation of blood vessel cells in human adaptive immune responses. Trends in Immunology 201233 49-57. (https://doi.org/10.1016/j.it.2011.09.006)

10 Cho I \& Blaser MJ. The human microbiome: at the interface of health and disease. Nature Reviews: Genetics 201213 260-270. (https://doi.org/10.1038/nrg3182)

11 Tang WHW, Li DY \& Hazen SL. Dietary metabolism, the gut microbiome, and heart failure. Nature Reviews: Cardiology 201916 137-154. (https://doi.org/10.1038/s41569-018-0108-7)

12 Foster JA, Rinaman L \& Cryan JF. Stress and the gut-brain axis: regulation by the microbiome. Neurobiology of Stress $2017 \mathbf{7} 124-136$. (https://doi.org/10.1016/j.ynstr.2017.03.001)

13 Dinan TG \& Cryan JF. Regulation of the stress response by the gut microbiota: implications for psychoneuroendocrinology. Psychoneuroendocrinology 201237 1369-1378. (https://doi. org/10.1016/j.psyneuen.2012.03.007)

14 Ehlers S, Kaufmann SHE \& Participants of the 99(th) Dahlem Conference. Infection, inflammation, and chronic diseases: consequences of a modern lifestyle. Trends in Immunology 201031 184-190. (https://doi.org/10.1016/j.it.2010.02.003)

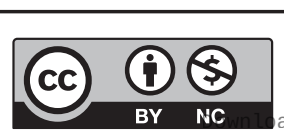

This work is licensed under a Creative Commons Attribution-NonCommercial 4.0 International License. 
15 Naviaux RK. Metabolic features of the cell danger response. Mitochondrion 201416 7-17. (https://doi.org/10.1016/j. mito.2013.08.006)

16 Tang WHW, Kitai T \& Hazen SL. Gut microbiota in cardiovascular health and disease. Circulation Research 2017120 1183-1196. (https://doi.org/10.1161/CIRCRESAHA.117.309715)

17 Janeway CA. Approaching the asymptote? Evolution and revolution in immunology. Cold Spring Harbor Symposia on Quantitative Biology 198954 1-13. (https://doi.org/10.1101/sqb.1989.054.01.003)

18 Van Linthout S, Miteva K \& Tschöpe C. Crosstalk between fibroblasts and inflammatory cells. Cardiovascular Research 2014102 258-269. (https://doi.org/10.1093/cvr/cvu062)

19 Travers JG, Kamal FA, Robbins J, Yutzey KE \& Blaxall BC. Cardiac fibrosis: the fibroblast awakens. Circulation Research 2016118 1021-1040. (https://doi.org/10.1161/CIRCRESAHA.115.306565)

20 Blyszczuk P, Müller-Edenborn B, Valenta T, Osto E, Stellato M, Behnke S, Glatz K, Basler K, Lüscher TF, Distler O, et al. Transforming growth factor- $\beta$-dependent Wnt secretion controls myofibroblast formation and myocardial fibrosis progression in experimental autoimmune myocarditis. European Heart Journal 201738 1413-1425. (https://doi.org/10.1093/eurheartj/ehw116)

21 Pober JS \& Sessa WC. Evolving functions of endothelial cells in inflammation. Nature Reviews: Immunology 20077 803-815. (https:// doi.org/10.1038/nri2171)

22 Aguilera G, Rabadan-Diehl C, Kiss A \& Ochedalski T. Vasoactive hormones and regulation of the hypothalamic-pituitary-adrenal axis. In Catecholamine Research. Kluwer Academic/Plenum Publishers, 2002. (https://doi.org/10.1007/978-1-4757-3538-3)

23 Alvino VV, Fernández-Jiménez R, Rodriguez-Arabaolaza I, Slater S, Mangialardi G, Avolio E, Spencer H, Culliford L, Hassan S, Sueiro Ballesteros L, et al. Transplantation of allogeneic pericytes improves myocardial vascularization and reduces interstitial fibrosis in a swine model of reperfused acute myocardial infarction. Journal of the American Heart Association 20187 e006727. (https://doi.org/10.1161/ JAHA.117.006727)

24 Spencer HL, Jover E, Cathery W, Avolio E, Rodriguez-Arabaolaza I, Thomas AC, Alvino VV, Sala-Newby G, Dang Z, Fagnano M, et al. Role of TPBG (trophoblast glycoprotein) antigen in human pericyte migratory and angiogenic activity. Arteriosclerosis, Thrombosis, and Vascular Biology 201939 1113-1124. (https://doi.org/10.1161/ ATVBAHA.119.312665)

25 Mangialardi G, Ferland-McCollough D, Maselli D, Santopaolo M, Cordaro A, Spinetti G, Sambataro M, Sullivan N, Blom A \& Madeddu P. Bone marrow pericyte dysfunction in individuals with type 2 diabetes. Diabetologia 201962 1275-1290. (https://doi. org/10.1007/s00125-019-4865-6)

26 Armulik A, Abramsson A \& Betsholtz C. Endothelial/pericyte interactions. Circulation Research 200597 512-523. (https://doi. org/10.1161/01.RES.0000182903.16652.d7)

27 Hirschi KK \& D'Amore PA. Pericytes in the microvasculature. Cardiovascular Research 199632 687-698.

28 Hulsmans M, Clauss S, Xiao L, Aguirre AD, King KR, Hanley A, Hucker WJ, Wülfers EM, Seemann G, Courties G, et al. Macrophages facilitate electrical conduction in the heart. Cell 2017169510. e20-522.e20. (https://doi.org/10.1016/j.cell.2017.03.050)

29 Baxter AG \& Hodgkin PD. No luck replicating the immune response in twins. Genome Medicine 20157 29. (https://doi.org/10.1186/ s13073-015-0156-0)
30 Dhabhar FS. Effects of stress on immune function: the good, the bad, and the beautiful. Immunologic Research 201458 193-210. (https:// doi.org/10.1007/s12026-014-8517-0)

31 Cho JH, Lee I, Hammamieh R, Wang K, Baxter D, Scherler K, Etheridge A, Kulchenko A, Gautam A, Muhie S, et al. Molecular evidence of stress-induced acute heart injury in a mouse model simulating posttraumatic stress disorder. PNAS 2014111 3188-3193. (https://doi.org/10.1073/pnas.1400113111)

32 Dal Lin C, Marinova M, Rubino G, Gola E, Brocca A, Pantano G, Brugnolo L, Sarais C, Cucchini U, Volpe B, et al. Thoughts modulate the expression of inflammatory genes and may improve the coronary blood flow in patients after a myocardial infarction. Journal of Traditional and Complementary Medicine 20188 150-163. (https://doi. org/10.1016/j.jtcme.2017.04.011)

33 Dal Lin C, Gola E, Brocca A, Rubino G, Marinova M, Brugnolo L, Plebani $\mathrm{M}$, Iliceto $\mathrm{S} \&$ Tona F. miRNAs may change rapidly with thoughts: the relaxation response after myocardial infarction. European Journal of Integrative Medicine 201820 63-72. (https://doi. org/10.1016/j.eujim.2018.03.009)

34 Aon MA. Systems Biology of Metabolic and Signaling Networks. Berlin, Heidelberg: Springer, 2014. (https://doi.org/10.1007/978-3-642-38505-6)

35 Bottaccioli F \& Bottaccioli AG. Psiconeuroendocrinoimmunologia e scienza della cura integrata. Il manuale. Edra 2017.

36 Fleshner M. Stress-evoked sterile inflammation, danger associated molecular patterns (DAMPs), microbial associated molecular patterns (MAMPs) and the inflammasome. Brain, Behavior, and Immunity 2013 27 1-7. (https://doi.org/10.1016/j.bbi.2012.08.012)

37 Chen GY \& Nuñez G. Sterile inflammation: sensing and reacting to damage. Nature Reviews: Immunology 201010 826-837. (https://doi. org/10.1038/nri2873)

38 Joshi H \& Morley SC. Cells under stress: the mechanical environment shapes inflammasome responses to danger signals. Journal of Leukocyte Biology 2019106 119-125. (https://doi. org/10.1002/JLB.3MIR1118-417R)

39 Saxton RA \& Sabatini DM. MTOR signaling in growth, metabolism, and disease. Cell 20179 960-976. (https://doi.org/10.1016/j. cell.2017.02.004)

$40 \mathrm{Li}$ X \& Stark GR. NFкB-dependent signaling pathways. Experimental Hematology 200230 285-296. (https://doi.org/10.1016/S0301472X(02)00777-4)

41 Picard M, McEwen BS, Epel ES \& Sandi C. An energetic view of stress: focus on mitochondria. Frontiers in Neuroendocrinology 201849 72-85. (https://doi.org/10.1016/j.yfrne.2018.01.001)

42 Macario AJ \& Conway de Macario E. Stress and molecular chaperones in disease. International Journal of Clinical and Laboratory Research 200030 49-66. (https://doi.org/10.1007/s005990070016)

43 Pick M, Flores-Flores C \& Soreq H. From brain to blood: alternative splicing evidence for the cholinergic basis of mammalian stress responses. Annals of the New York Academy of Sciences 20041018 85-98. (https://doi.org/10.1196/annals.1296.010)

44 Babenko O, Kovalchuk I \& Metz GAS. Stress-induced perinatal and transgenerational epigenetic programming of brain development and mental health. Neuroscience and Biobehavioral Reviews 201548 70-91. (https://doi.org/10.1016/j.neubiorev.2014.11.013)

45 Guerrero-Bosagna C \& Skinner MK. Environmentally induced epigenetic transgenerational inheritance of phenotype and disease. Molecular and Cellular Endocrinology 2012354 3-8. (https://doi. org/10.1016/j.mce.2011.10.004)

Received in final form 12 August 2019

Accepted 15 August 2019

Accepted Preprint published online 15 August 2019 https://vb.bioscientifica.com

https://doi.org/10.1530/VB-19-0023 (c) 2019 The authors Published by Bioscientifica Ltd

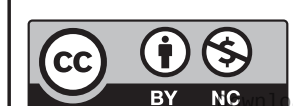

This work is licensed under a Creative Commons Attribution-NonCommercial 4.0 International License. ded from Bioscientifica com at 04/26/2023 08:45:05AM 